\title{
Agreement between Maternal Cannabis Use during Pregnancy according to Self-Report and Urinalysis in a Population-Based Cohort: The Generation R Study
}

\author{
H. El Marroun ${ }^{a, b}$ H. Tiemeier ${ }^{c, d}$ V.W.V. Jaddoe ${ }^{a, c, d} \quad$ A. Hofman ${ }^{c}$ F.C. Verhulst ${ }^{b}$ \\ W. van den Brinke, f A.C. Huizink ${ }^{b, g}$ \\ ${ }^{a}$ The Generation R Study Group, Erasmus Medical Centre Rotterdam, Rotterdam, ${ }^{b}$ Department of Child and \\ Adolescent Psychiatry, Erasmus Medical Centre, Sophia Children's Hospital, Rotterdam, 'Department of \\ Epidemiology, Erasmus Medical Centre Rotterdam, Rotterdam, dDepartment of Paediatrics, Erasmus Medical \\ Centre Rotterdam, Rotterdam, ${ }^{\mathrm{e}}$ Academic Medical Centre University of Amsterdam, Amsterdam, ${ }^{\mathrm{f}} \mathrm{Amsterdam}$ \\ Institute for Addiction Research, Amsterdam, and ${ }^{9}$ Department of Education, Faculty of Behavioural and Social \\ Sciences, University of Amsterdam, Amsterdam, The Netherlands
}

\section{Key Words}

Cannabis · Self-report $\cdot$ Pregnancy $\cdot$ Urine testing

\begin{abstract}
Aim: To verify self-reported information on prenatal drug use in urine because reporting in pregnancy is sensitive to stigma and might lead to misclassification. Methods: Using semiquantitative immunochemical analysis, the presence of the urinary metabolite (11-nor- $\Delta 9$-tetrahydrocannabinol9-carboxylic acid) was compared to self-reported prenatal cannabis use. Sensitivity and specificity for self-report and urinalysis outcomes were calculated and Yule's $Y$ was used as an agreement measure. Results: Urine samples were available for 3,997 pregnant women. Of these women, 92 reported having used cannabis during pregnancy (2.3\%) and 71 had positive urine screens (1.8\%). In total $35 \%$ of the 92 women with self-reported cannabis use also had a positive urine screen. Positive urines were relatively frequent in women reporting cannabis use before pregnancy only (7.6\%) and in women with missing information (2.6\%). Sensitivity and specificity of urinalysis compared to self-report were 0.46 and 0.98 . Sensitivity and specificity of self-report compared to urinalysis were 0.36 and 0.99 . Yule's $Y$ amounted to 0.77 ,
\end{abstract}

indicating substantial agreement between the measures. Conclusions: Our findings illustrate the difficulties in obtaining valid information on prenatal cannabis use. To improve the quality of cannabis use data, we suggest a 2-step approach starting with self-report.

Copyright $\odot 2010$ S. Karger AG, Basel

\section{Introduction}

Cannabis is the most commonly used illicit substance in Western countries. In Europe, the 12-month prevalence of cannabis use among young adults increased from $5 \%$ in 1990 to $15 \%$ in 2005 [1]. In young Australian women, the 12 -month prevalence of cannabis use was $24.4 \%$ [2], and in young Canadian women it was $21.8 \%$ [3]. In pregnant women, the prevalence of cannabis use was much lower, i.e. in 2007, 2.9\% of Dutch women and $1.8 \%$ of American women reported prenatal cannabis use $[4,5]$.

In epidemiological studies, researchers often use different methods to assess substance use, such as biochemical measures and self-report. Biochemical measures provide clear information on recent substance use, yet are

\section{KARGER}

Fax +41613061234 E-Mail karger@karger.ch www.karger.com
(C) 2010 S. Karger AG, Basel

1022-6877/11/0171-0037\$38.00/0

Accessible online at:

www.karger.com/ear
Hanan El Marroun

Department of Child and Adolescent Psychiatry

Erasmus MC, Sophia Children's Hospital, PO Box 2060

NL-3000 CB Rotterdam (The Netherlands)

Tel. +31 107034 275, Fax +31 107032 1111, E-Mail h.marrounel@erasmusmc.nl 
restricted by error rates, brief detection time periods and high costs. Self-reports are less invasive and permit the evaluation of substance use over longer periods in time, but are influenced by possible reporter social desirability and forgetfulness. Although multiple studies on consistency and validity of multiple assessment methods among adults and adolescents have been reported [6], little information is available on the agreement between self-reported cannabis use and urinalysis in pregnancy. Previous hospital-based research has demonstrated that self-reported use correlates moderately with biochemical measures of exposure to cannabis $[7,8]$.

In previous studies of a population-based cohort in the Netherlands, we based prenatal cannabis use on self-reported information collected using a questionnaire in pregnancy $[4,9]$. Although self-report is a commonly used measure to assess substance use, it is acknowledged that individuals may underreport or deny substance use, especially during pregnancy [10]. Cannabis use is not prosecuted in the Netherlands, and false negative reporting may therefore occur less frequently than in other countries; however, misclassification cannot be ruled out. Factors that may lead pregnant women to underreport substance use could be social desirability, forgetfulness, perceived norms about acceptability and fear of consequences. Therefore, it is important to verify self-reported information on prenatal cannabis use by means of detecting urinary cannabis metabolites. Moreover, we were particularly interested in exploring whether or not missing information on self-reported cannabis use was related to an increased risk of positive urine screens. In this study, the focus is on prenatal cannabis use exclusively, as in a general population-based cohort, the prevalence of other illicit drug use during pregnancy (e.g. cocaine, amphetamines) is expected to be very low. In addition, urinary detection time of other substances is shorter (e.g. cocaine assesses 1-3 days) as compared to detection time of the cannabis metabolite, which has a long half-life in the body of up to several days or even weeks, depending on the frequency of use [11-15].

In the current study, self-reported information on prenatal cannabis use was compared tothe presence of the metabolite 11-nor- $\Delta 9$-tetrahydrocannabinol-9-carboxylic acid (11-nor- $\Delta$ 9-THC-9-COOH) using semiquantitative immunochemical urinalysis. Although available data suggest that self-reported cannabis use correlates only moderately with biochemical measures, we hypothesize substantial agreement between these measures in our cohort as cannabis use is not prosecuted in the Netherlands [16].

\section{Methods}

\section{Study Population}

This study was conducted within the Generation R study, a population-based birth cohort in Rotterdam, the Netherlands [17], set up to collect data on a sample of parents and their children from early pregnancy onwards. All children were born between April 2002 and January 2006 and constitute a prenatally enrolled birth cohort that is currently followed until young adulthood. In total 8,880 women (response rate $61 \%$ ) were enrolled in Generation R during pregnancy. The study was conducted in accordance with the guidelines proposed in the World Medical Association Declaration of Helsinki, and it was approved by the Medical Ethics Committee of the Erasmus Medical Centre, Rotterdam. Written informed consent was obtained from all participants.

\section{Self-Reported Maternal Substance Use}

Alcohol, tobacco and cannabis use were measured using a selfreport questionnaire at enrolment (usually in the 1st trimester of pregnancy; response rate for report on cannabis use was $85.7 \%$ ). These questionnaires were handled anonymously by using barcodes instead of names and birthdates. We explicitly asked them to retrospectively reply to 2 separate questions of whether they had used these substances before pregnancy, and whether they had used them in the last 3 months. In the latter question the response options were: 'no', 'yes, until I knew I was pregnant' and 'yes, I still use substances'. This information was therefore not specific for the entire gestational period. Mothers also provided information on the substance use of the biological father of the child. Based on these questions, we grouped the total population ( $\mathrm{n}=8,880$ ) for this study in 4 nonoverlapping categories: (1) cannabis use before and during pregnancy $(\mathrm{n}=220),(2)$ cannabis use before pregnancy only $(\mathrm{n}=246)$, (3) missing information on cannabis use $(\mathrm{n}=1,270)$ and (4) no cannabis use before or during pregnancy $(n=7,144)$. Self-reported numbers of cannabis use before pregnancy $(2.8 \%)$ and during pregnancy $(2.5 \%)$ were in agreement with national numbers of cannabis use among Dutch women aged between 15 and 64 years (recent use: $3.1 \%$; current use: $1.5 \%$ ) in the same period [18]. Substance use was unknown for 1,270 women of the total population (14.3\%), which was due to failure to return the questionnaire $(n=764)$ or failure to fill out the specific question on substance use $(n=506)$.

\section{Urine Collection and Analysis}

Details of biological specimen collection and storage have been described previously [17]. Maternal urine samples were collected in early, mid- and late pregnancy between February 2004 and November 2005. From April to July 2008, about 14 batches ( $\mathrm{n}=300$ per batch) of $3 \mathrm{ml}$ of urine were sent to the Delta laboratory in Poortugaal, the Netherlands, and were tested on the presence of 11-nor- $\Delta$ 9-THC-9-COOH using the DRI ${ }^{\circledR}$ Cannabinoid Assay (Microgenics) with a cutoff value of $50 \mu \mathrm{g} / \mathrm{l}$ as recommended by the manufacturer and the Substance Abuse and Mental Health Security Agency. Using lower cutoff levels (20-40 $\mu \mathrm{g} / \mathrm{l})$ resulted in similar values of agreement between urinalyses and self-reports. We therefore only reported the results using the recommended cutoff level of $50 \mu \mathrm{g} / \mathrm{l}$. For each urine sample, creatinine concentrations were determined, as this provides information on possible urinary dilution (normal urine $\geq 1.8 \mathrm{mmol} / \mathrm{l}$; diluted $<1.8 \mathrm{mmol}$; inconsistent with human urine $\leq 0.4 \mathrm{mmol} / \mathrm{l}$ ) 
$[19,20]$. Of all samples, $5.6 \%(n=222)$ were diluted, and based on the creatinine levels only 1 sample was deemed as inconsistent with human urine. Deleting or including these samples in the analyses did not change any of the results. Urine samples were missing in almost half of the cohort because urine sample collection was performed during a limited period in the prenatal phase of the study [17]. For the current study, we could therefore use urine samples of 3,997 pregnant women. Due to this limited period of urine collection, $78.9 \%(n=2,375)$ of the pregnant women filled out the questionnaire after urine collection, and the remaining $21.1 \%$ filled out the questionnaire before urine sample collection.

\section{Data Analysis}

First, we compared women with urine samples $(\mathrm{n}=3,997)$ and women without urine samples $(n=4,883)$ on several characteristics such as maternal age, ethnicity [21], educational level [22], maternal psychopathology [23] and maternal report on alcohol, tobacco and cannabis use during pregnancy using independent $t$ tests for continuous variables and a $\chi^{2}$ test for categorical variables. The urinary average creatinine levels were compared using ANOVAs among the following groups: women reporting (1) cannabis use during pregnancy, (2) cannabis use before pregnancy, (3) no cannabis use and (4) women without information on cannabis use. Self-reported data on cannabis use and the presence of 11-nor- $\Delta$ 9-THC-9-COOH in maternal urine were compared using a $2 \times 2$ contingency table. We calculated the sensitivity and specificity for urinalyses compared to self-report, and vice versa, because we did not consider either of the 2 measurements as the golden standard.

Additionally, Yule's Y was calculated as a measure of overall agreement between self-reported cannabis use and urinalysis findings. Yule's $Y$, also called the coefficient of colligation for dichotomous variables, is exactly equivalent to Hoehler's adjusted $\kappa$ of agreement [24], but appears to be less dependent on prevalence than $к$. Yule's $Y$ is a symmetric measure taking on values between -1 and +1 implying a perfect negative or positive association 0 implies no association. Yule's $\mathrm{Y}$ is calculated using the $2 \times 2$ table with: $\mathrm{Y}=(\sqrt{\mathrm{ad}}-\sqrt{\mathrm{bc}}) /(\sqrt{\mathrm{ad}}+\sqrt{\mathrm{bc}})$. Results were considered significant at $\mathrm{p}<0.01$. We chose this conservative significance level because given a sufficiently large sample, as we have in this study, extremely small and negligible differences can be found to be statistically significant. Statistical analyses were performed using the Statistical Package of Social Sciences version 15.0 for Windows (SPSS Inc., Chicago, Ill., USA).

\section{Results}

\section{Attrition Analysis}

The urine samples of 3,997 of 8,880 pregnant mothers were available (table 1). Nonresponse analyses showed small differences between women with and without urine samples; women without urine samples were significantly younger ( 0.5 years), somewhat lower educated $(39.4 \%$ higher educated vs. $45.5 \%)$ and smoked slightly more often during pregnancy ( 25.7 vs. $22.2 \%$ ) as compared to
Table 1. Characteristics of mothers with and without urine samples

\begin{tabular}{|c|c|c|c|}
\hline & $\begin{array}{l}\text { Mothers with } \\
\text { urine samples } \\
(\mathrm{n}=3,997)\end{array}$ & $\begin{array}{l}\text { Mothers without } \\
\text { urine samples } \\
(\mathrm{n}=4,883)\end{array}$ & $\begin{array}{l}\text { Effect } \\
\text { size }\end{array}$ \\
\hline Maternal age & $29.9 \pm 5.2$ & $29.4 \pm 5.4^{*}$ & 0.10 \\
\hline \multicolumn{4}{|l|}{ Maternal education, \% } \\
\hline Primary education & 12.3 & $13.3^{*}$ & \multirow[t]{3}{*}{0.06} \\
\hline Secondary education & 42.3 & 47.3 & \\
\hline Higher education & 45.5 & 39.4 & \\
\hline \multicolumn{4}{|l|}{ Marital status, \% } \\
\hline Nonmarried & 49.4 & 51.9 & 0.03 \\
\hline \multicolumn{4}{|l|}{ Maternal psychopathology } \\
\hline General Symptom Index & $0.29 \pm 0.38$ & $0.31 \pm 0.39$ & 0.05 \\
\hline \multicolumn{4}{|c|}{ Maternal ethnicity, \% } \\
\hline Dutch & 48.2 & 50.3 & \multirow[t]{2}{*}{0.02} \\
\hline Non-Dutch & 51.8 & 49.4 & \\
\hline \multicolumn{4}{|l|}{ Maternal tobacco use, $\%$} \\
\hline None & 61.3 & $56.9^{*}$ & \multirow[t]{3}{*}{0.05} \\
\hline Before pregnancy & 16.5 & 17.4 & \\
\hline During pregnancy & 22.2 & 25.7 & \\
\hline \multicolumn{4}{|l|}{ Maternal alcohol use, \% } \\
\hline None & 33.1 & 32.5 & \multirow[t]{3}{*}{0.02} \\
\hline Before pregnancy & 24.8 & 24.5 & \\
\hline During pregnancy & 42.1 & 43.1 & \\
\hline \multicolumn{4}{|l|}{ Maternal cannabis use, $\%$} \\
\hline None & 80.1 & 80.7 & \multirow[t]{4}{*}{0.02} \\
\hline Before pregnancy & 3.0 & 2.6 & \\
\hline During pregnancy & 2.3 & 2.6 & \\
\hline Unknown & 14.7 & 14.0 & \\
\hline \multicolumn{4}{|l|}{ Paternal cannabis use, $\%$} \\
\hline None & 88.7 & 87.7 & \multirow[t]{3}{*}{0.02} \\
\hline Yes & 10.2 & 10.7 & \\
\hline Unknown & 1.2 & 1.6 & \\
\hline
\end{tabular}

Figures are mean $\pm \mathrm{SD}$ or percentages. Statistical significance was derived from independent $t$ tests for continuous variables and a $\chi^{2}$ test for categorical variables $\left({ }^{*} p<0.01\right)$. Effects sizes were calculated by Cohen's $\mathrm{d}$ for continuous variables and phi was used for categorical variables.

women with urine samples. No statistically significant differences in maternal and paternal cannabis use, maternal alcohol use and ethnicity were found.

\section{Self-Report and Urinalysis}

Of 3,997 pregnant women, 92 reported having used cannabis during pregnancy $(2.3 \%)$, while we found 71 positive urine screens of all women whether they reported cannabis use or not (1.8\%). The numbers (92 self-reports and 71 positive urine screens) were not completely overlapping (table 2). Of the 92 mothers who reported having used cannabis during pregnancy, 33 mothers had 
Table 2. $2 \times 2$ contingency table for calculating sensitivity, specificity and Yule's Y with absolute and relative numbers of available urine samples, positive detected urine screens and mean creatinine level

\begin{tabular}{|c|c|c|c|c|}
\hline \multirow[t]{2}{*}{ Maternal self-report } & \multicolumn{4}{|l|}{ Urinalysis } \\
\hline & positive $(\%)^{\mathrm{a}}$ & negative $(\%)^{\mathrm{a}}$ & total $(\%)^{\mathrm{b}}$ & $\begin{array}{l}\text { creatinine in } \mathrm{mmol} / \mathrm{l} \\
(\text { mean } \pm \mathrm{SD})\end{array}$ \\
\hline Cannabis use during pregnancy (Group A; $\mathrm{n}=220)$ & $33(35.9)$ & $59(64.1)$ & $92(41.8)$ & $9.4 \pm 5.5$ \\
\hline All other women (Group B; $\mathrm{n}=8,660)$ & $38(1.0)$ & $3,867(99.0)$ & $3,905(45.1)$ & $8.4 \pm 5.4$ \\
\hline Total $(\mathrm{n}=8,880)$ & $71(1.8)$ & $3,926(98.2)$ & $3,997(45.0)$ & $8.4 \pm 5.4$ \\
\hline Cannabis use during pregnancy (Group A; $\mathrm{n}=220)$ & $33(35.9)$ & $59(64.1)$ & $92(41.8)$ & $9.4 \pm 5.5$ \\
\hline Cannabis use before pregnancy (Group $\left.B_{1} ; n=246\right)$ & $9(7.6)$ & $109(92.4)$ & $118(47.9)$ & $8.6 \pm 4.2$ \\
\hline Missing information (Group $\mathrm{B}_{2} ; \mathrm{n}=1,270$ ) & $15(2.6)$ & $571(556)$ & $586(46.1)$ & $8.8 \pm 5.0$ \\
\hline No cannabis use during pregnancy $\left(\right.$ Group $\left.B_{3} ; n=7,144\right)$ & $14(0.4)$ & $3,187(99.6)$ & $3,201(44.8)$ & $8.4 \pm 5.5$ \\
\hline Total $(\mathrm{n}=8,880)$ & $71(1.8)$ & $3,926(98.2)$ & $3,997(45.0)$ & $8.4 \pm 5.4$ \\
\hline
\end{tabular}

Group $B$ consists of 3 subgroups: $\mathrm{B}_{1}$, women reporting cannabis before pregnancy; $\mathrm{B}_{2}$, women without information on cannabis use; and $\mathrm{B}_{3}$, women reporting no cannabis use.

${ }^{a}$ Proportion of positive and negative urine screens based on the number of urine samples.

b Proportion of urine samples based on the total number of participants.

a positive urine screen. Based on these data, the total number of women with at least some evidence of cannabis use during pregnancy amounts to 92 (self-reported) + $15+14+9$ (positive urine tests without self-reported cannabis use) $=130$, i.e. $3.3 \%$ of the group with urinalysis data (table 2, lower part). And, these data indicate that 92 of the 130 women with probable cannabis use during pregnancy $(70.7 \%)$ reported their cannabis use. In addition, based on a combination of positive self-report and positive urinalysis data, at least 130 of the 3,997 pregnant women used cannabis during pregnancy (3.3\%), i.e. 1.43 times the prevalence of cannabis use during pregnancy based on self-reported data only and 1.83 times the prevalence based on urinalysis data only. Table 2 (lower part) demonstrates that a sizeable proportion of positive urine screens was found in women who reported cannabis use during pregnancy (35.9\%), a substantial proportion of positive screens was found in women who reported cannabis use only before pregnancy (7.6\%), a very small proportion of positive screens was found in women who reported no cannabis use (0.4\%), and an intermediate proportion of positive screens was found in women without self-report data on cannabis use (2.6\%).

\section{Creatinine Levels}

Average creatinine levels were not statistically different across groups $(\mathrm{F}=2.23, \mathrm{p}=0.082$; table 2$)$. Moreover, no significant difference between mean maternal urinary creatinine levels in women who reported having used cannabis during pregnancy $(9.4 \pm 5.5 \mathrm{mmol} / \mathrm{l})$ and of mothers who reported not having used cannabis during pregnancy $(8.4 \pm 5.4 \mathrm{mmol} / \mathrm{l})$ was found $(\mathrm{t}=1.78 ; \mathrm{p}=$ 0.074). Mean creatinine levels for cannabinoid-positive $(\mathrm{n}=71, \mu=12.5 \pm 7.0 \mathrm{mmol} / \mathrm{l})$ and cannabinoid-negative urine samples $(\mathrm{n}=3,926, \mu=8.4 \pm 5.3 \mathrm{mmol} / \mathrm{l})$ were significantly different $(\mathrm{t}=6.41 ; \mathrm{p}<0.001)$.

\section{Sensitivity, Specificity and Yule's $Y$}

Sensitivity and specificity of the urinalysis as compared to self-report were $33 / 71=0.46$ and 3,867/3,926= 0.98 , respectively. In addition, sensitivity and specificity of self-report as compared to urinalysis were $33 / 92=0.36$ and 3,867/3,905 $=0.99$. These findings (based on table 2) indicate that both approaches perform very well in the identification of noncannabis users, but that both measures seem to identify partially different subpopulations of cannabis users during pregnancy. Based on table 2, it was possible to calculate overall Yule's Y: $(\sqrt{33} \cdot 3,867-$ $\sqrt{59} \cdot 38) /(\sqrt{33} \cdot 3,867+\sqrt{59} \cdot 38)=0.77$, indicating substantial agreement between the 2 measures. When calculated by comparing pregnant cannabis users and women who reported nonuse (Group $\mathrm{B}_{3}$ in table 2), Yule's $\mathrm{Y}$ was somewhat higher: $(\sqrt{33} \cdot 3,187-\sqrt{59 \cdot 14}) /(\sqrt{33} \cdot 3,187+\sqrt{59 \cdot 14})=$ 0.84 . 


\section{Paternal Cannabis Use}

Additional analyses showed that, compared to women that reported not using cannabis, among pregnant women that reported cannabis use, paternal cannabis use was more common (78.9 vs. $8.5 \%$; $<<0.001$; phi $=0.38$ ). Moreover, paternal cannabis use was also more common when maternal urine samples were positive (71.7 vs. $9.2 \%$; $p<$ 0.001; phi $=0.26$ )

\section{Discussion}

In this study, we compared self-reported information on prenatal maternal cannabis use with the presence of the cannabis metabolite, 11-nor-9-THC-9-COOH, measured by semiquantitative immunochemical urinalysis. Our findings demonstrated that reliance on urinalysis of a single urine specimen underestimates the prevalence of cannabis use during pregnancy profoundly and may be biased toward long-term or heavy users, as they are more likely than occasional users to be detected through urinalysis. However, reliance on self-reported cannabis alone also underestimates the prevalence of prenatal cannabis use even in the Netherlands, where neither cannabis possession nor cannabis use is prosecuted.

Our findings are to some extent in agreement with other studies. First, our findings are consistent with the report by Shiono et al. [7] that only $43.2 \%$ of the women reporting cannabis also had a positive serum assay result. However, our findings were not fully consistent with the data of Markovic et al. [8], who reported much higher proportions of positive urine screens (86.6\%) in women reporting current cannabis use in early pregnancy. A plausible explanation for this finding is that this sample was not representative of the general population, but was a selected group of 570 (out of 1,347) pregnant women with a high prevalence of cannabis use during pregnancy measured by self-report (16.8\%) and urinalysis (21.5\%) [25]. Previous studies with different populations have reported moderate agreement between self-reports on cannabis use and urinalysis [6, 26]; thus, we reported a higher agreement between self-report of cannabis use during pregnancy and urinalysis.

Sensitivity, of both self-report and urinalysis, was moderate, whereas specificity of both assessment procedures was high. If it can be assumed that false positive self-reports for cannabis use are unlikely, the negative urine screens of pregnant women reporting cannabis use during pregnancy are of interest. The main metabolite excreted in the urine (11-nor- $\Delta$ 9-THC-9-COOH) is found within hours of exposure and remains detectable in the urine for 3-10 days after smoking a single dose. However, the length of time following cannabis use for a positive urine screen is dependent upon multiple factors, including the frequency and amount of cannabis, metabolic rate, excretion rate, half-life time, storage of the urine samples and the cannabis-user's age, body fat content, activity and diet $[27,28]$. These difficulties may explain the finding of negative urine screens in 59 of the 92 women (64\%) who reported cannabis use during pregnancy. A possible reason for this, and probably the main limitation of this study, is that we only took 1-3 urine samples per individual throughout pregnancy. Ideally, weekly urine sampling would provide better estimates and would provide information about newly used cannabis as well. So, in large epidemiological studies with limited numbers of urine samples, immunochemical urinalysis performed once per trimester cannot be regarded as the golden standard in assessing prenatal cannabis use. This may also mean that in the group of mothers who did not answer the question about substance use or reported nonuse, the true prevalence of cannabis use may be even higher. In fact, if the prevalence of prenatal cannabis use were estimated based on the urinalysis findings, the fact that at least two thirds of cannabis-using women were not detected in our study with urinalysis should be taken into account.

In addition, some other limitations in this study should be considered. First, extended frozen storage may have possibly led to degradation of cannabinoids over time, and may have led to an underestimation of the prevalence of cannabinoids in urine. However, several studies have shown that change in concentrations of 11-nor- $\Delta 9$-THC9- $\mathrm{COOH}$ after long-term storage was not extensive $(<15 \%)[29,30]$. Second, we cannot rule out that women may wonder about specific aims for urine collection. We expected that knowing urine samples were collected (without a specific reason) did not influence cannabis use among these women. Third, exact information on the amount of time that had elapsed between filling out the questionnaire and urine collection was not available; however, in general women who visited the research center for anthropometrics, ultrasound measurements and blood and urine collection filled out the questionnaire at approximately the same time. Alternative detection methods such as hair examination is limited too, as cannabis incorporation depends on growth rate, anatomical region, age, gender, ethnicity, hair color and individual variability [31]. One could consider testing cannabis by using meconium, but meconium testing has some limita- 
tions as well. First, meconium begins forming in the 12th week of gestation, so this theoretically means collecting endogenous and exogenous wastes from the 2 nd trimester onwards [32]. Therefore, in large populations with limited resources, the most sensible method is to use selfreported information on prenatal drug exposure, as this is likely to provide the best estimate for the lowest price (highest cost effectiveness). Fourth, the nonresponse analysis showed small differences between women with and without urine samples, which may increase the likelihood for cannabis use in women without urine samples. However, we previously reported that paternal cannabis use is the main determinant for prenatal cannabis use [4], and in the current study, the additional analyses showed that paternal cannabis use occurs more often when mothers report cannabis use during pregnancy or when cannabinoids are detected in urine samples. Therefore, it is essential to consider paternal cannabis use. Finally, a total of $61 \%$ of all eligible women participated in the Generation R study [33] and they may not be completely representative of the general Rotterdam population [34]. This may perhaps have led to an unfavorable selection of non- using women. Yet, this selection bias does not necessarily mean that the relationship between self-reported cannabis use and urinalysis presented in this study was distorted.

In conclusion, researchers and clinicians, should acknowledge that pregnant women may underreport current cannabis use, a situation that seems most prevalent in women admitting past cannabis use (i.e. cannabis use before the pregnancy) and in women refusing to provide information on prenatal cannabis use. Finally, our findings illustrate the difficulties in obtaining valid information on prenatal cannabis use. Nonetheless, selfreport seems to be an acceptable single method to determine cannabis use during pregnancy in epidemiological studies. Importantly, in order to improve the quality of cannabis use data, we suggest a 2-step approach starting with self-report information and followed by urinalysis in women who reported cannabis use before pregnancy, refused to answer questions about substance use during pregnancy and have partners that use cannabis.

\section{References}

1 European Monitoring Centre of Drugs and Drug Addiction: Annual Report 2006: The State of the Drugs Problem in Europe. Lisbon, EMCDDA, 2006.

-2 Turner C, Russell A, Brown W: Prevalence of illicit drug use in young Australian women, patterns of use and associated risk factors. Addiction 2003;98:1419-1426.

-3 Leatherdale ST, Hammond DG, Kaiserman M, Ahmed R: Marijuana and tobacco use among young adults in Canada: are they smoking what we think they are smoking? Cancer Causes Control 2007;18:391-397.

-4 El Marroun H, Tiemeier H, Jaddoe VW, Hofman A, Mackenbach JP, Steegers EA, Verhulst FC, van den Brink W, Huizink AC: Demographic, emotional and social determinants of cannabis use in early pregnancy: the Generation R study. Drug Alcohol Depend 2008;98:218-226.

-5 Ebrahim SH, Gfroerer J: Pregnancy-related substance use in the United States during 1996-1998. Obstet Gynecol 2003;101:374379.

-6 Buchan BJ, M LD, Tims FM, Diamond GS: Cannabis use: consistency and validity of self-report, on-site urine testing and laboratory testing. Addiction 2002;97(suppl 1):98108
7 Shiono PH, Klebanoff MA, Nugent RP, Cotch MF, Wilkins DG, Rollins DE, Carey JC, Behrman RE: The impact of cocaine and marijuana use on low birth weight and preterm birth: a multicenter study. Am J Obstet Gynecol 1995;172:19-27.

-8 Markovic N, Ness RB, Cefilli D, Grisso JA, Stahmer S, Shaw LM: Substance use measures among women in early pregnancy. Am J Obstet Gynecol 2000;183:627-632.

9 El Marroun H, Tiemeier H, Steegers EA, Jaddoe VW, Hofman A, Verhulst FC, van den Brink W, Huizink AC: Intrauterine cannabis exposure affects fetal growth trajectories: the Generation R study. J Am Acad Child Adolesc Psychiatry 2009, E-pub ahead of print.

10 Jacobson SW, Jacobson JL, Sokol RJ, Martier SS, Ager JW, Kaplan MG: Maternal recall of alcohol, cocaine, and marijuana use during pregnancy. Neurotoxicol Teratol 1991; 13:535-540

11 Cary PL: The marijuana detection window: determining the length of time cannabinoids will remain detectable in urine following smoking: a critical review of relevant research and cannabinoid detection guidance for drug courts. Drug Court Rev 2005;5:2358
-12 Huestis MA, Cone EJ: Urinary excretion half-life of 11-nor-9-carboxy-delta9-tetrahydrocannabinol in humans. Ther Drug Monit 1998;20:570-576

13 Kelly P, Jones RT: Metabolism of tetrahydrocannabinol in frequent and infrequent marijuana users. J Anal Toxicol 1992;16:228-235.

14 Fraser AD, Worth D: Urinary excretion profiles of 11-nor-9-carboxy-delta9-tetrahydrocannabinol: a delta9-thc-cooh to creatinine ratio study \#2. Forensic Sci Int 2003;133:2631.

15 Johansson E, Halldin MM: Urinary excretion half-life of delta 1-tetrahydrocannabinol-7-oic acid in heavy marijuana users after smoking. J Anal Toxicol 1989;13:218-223.

16 van den Brink W: Forum: decriminalization of cannabis. Curr Opin Psychiatry 2008;21: 122-126.

17 Jaddoe VW, Bakker R, van Duijn CM, van der Heijden AJ, Lindemans J, Mackenbach JP, Moll HA, Steegers EA, Tiemeier H, Uitterlinden AG, Verhulst FC, Hofman A: The Generation R Study Biobank: a resource for epidemiological studies in children and their parents. Eur J Epidemiol 2007;22:917-923.

18 Rodenburg G, Spijkerman R, Van den Eijnden R, Van de Mheen, D: Nationaal Prevalentie Onderzoek naar Middelengebruik 2005. Rotterdam, IVO, 2007. 
-19 Cook JD, Caplan YH, LoDico CP, Bush DM: The characterization of human urine for specimen validity determination in workplace drug testing: a review. J Anal Toxicol 2000;24:579-588.

20 Heit HA, Gourlay DL: Urine drug testing in pain medicine. J Pain Symptom Manage 2004;27:260-267.

21 Statistics Netherlands: Migrants in the Netherlands 2004 (Allochtonen in Nederland 2004), Voorburg/Heerlen, 2004. http: www.cbs.nl.

22 Statistics Netherlands: Standard Classification of Education 2003 (Standaard Onderwijsindeling 2003). Voorburg/Heerlen, 2004, http://www.cbs.nl.

-23 Derogatis LR, Melisaratos N: The brief symptom inventory: an introductory report. Psychol Med 1983;13:595-605.

24 Walter SD: Hoehler's adjusted kappa is equivalent to Yule's Y. J Clin Epidemiol 2001; 54:1072-1073.
25 Ness RB, Grisso JA, Hirschinger N, Markovic N, Shaw LM, Day NL, Kline J: Cocaine and tobacco use and the risk of spontaneous abortion. N Engl J Med 1999;340:333-339.

-26 Perrone J, De Roos F, Jayaraman S, Hollander JE: Drug screening versus history in detection of substance use in ED psychiatric patients. Am J Emerg Med 2001;19:49-51.

27 Rouen D, Dolan K, Kimber J: A review of drug detection testing and an examination of urine, hair, saliva and sweat. University of New South Wales, Sydney, Technical Report No. 120. Sydney, National Drug and Alcohol Research Centre, 2001.

28 Musshoff F, Madea B: Review of biologic matrices (urine, blood, hair) as indicators of recent or ongoing cannabis use. Ther Drug Monit 2006;28:155-163.

29 Dugan S, Bogema S, Schwartz RW, Lappas NT: Stability of drugs of abuse in urine samples stored at -20 degrees C. J Anal Toxicol 1994;18:391-396.

\$3 Moody DE, Monti KM, Spanbauer AC: Long-term stability of abused drugs and antiabuse chemotherapeutical agents stored at -20 degrees C. J Anal Toxicol 1999;23:535540 .
31 Wennig R: Potential problems with the interpretation of hair analysis results. Forensic Sci Int 2000;107:5-12.

32 Gray TR, LaGasse LL, Smith LM, Derauf C, Grant P, Shah R, Arria AM, Della Grotta SA, Strauss A, Haning WF, Lester BM, Huestis MA: Identification of prenatal amphetamines exposure by maternal interview and meconium toxicology in the Infant Development, Environment and Lifestyle (IDEAL) study. Ther Drug Monit 2009;31:769-775.

33 Jaddoe VW, Mackenbach JP, Moll HA, Steegers EA, Tiemeier H, Verhulst FC, Witteman JC, Hofman A: The Generation R study: design and cohort profile. Eur J Epidemiol 2006;21:475-484.

34 Central Bureau of Research and Statistics Rotterdam: Social structure Rotterdam (in Dutch). Rotterdam, Central Bureau of Research and Statistics Rotterdam (COS), 2003. 